\title{
Synthesis and Characterization of New-2,3-Disubstituted Quinazolinone Derivatives as Antibacterial Agents
}

\author{
Souad J. Lfta, ${ }^{1}$ Nabeel B. Ayram and Salah M. Baqer \\ Department of Chemistry, College of Sciences, University of Mustansiriya, Baghdad-Iraq. \\ ${ }^{1}$ E-mail: nabel_org@yahoo.com.
}

\begin{abstract}
In this work, some new 2,3-disubsituted quinazolinone derivatives were synthesized. The reaction of substituted anthranilic acid with 3-chlorophenylisothiocyanate gave the compounds [3-(3-chlorophenyl)-2-mercaptoquinazoline-4(3H)-one] (1a,b). compounds (1a,b) where treated with chloroethylacetate in presence of potassium carbonate gave the compounds [-4-oxo-3, 4-dihydroquinazoline-2-yl)thio)acetate] (2a,b). Reaction with hydrazine hydrate afforded the acetohydrazides [-4-oxo-3,4-dihydroquinazoline-2-yl) thio) acetohydrazide](3a,b). The acetoohydrazides $(3 a, b)$ were treated with aromatic aldehydes to give the Schiff's bases [-N ${ }^{-}-(3-$ nitrobenzylidene)acetohydrazide] $(5 a, b)$. Treatment of derivatives $(1 a, b)$ with hydrazine hydrate afforded the hydrazine derivatives [-2-hydrazinylquinazoilne-4(3H)-one] $(4 \mathrm{a}, \mathrm{b})$ which also used in synthesizing Schiff's bases [(E)(2-(3-nitrobenzyllidene)hydrazinyl)] (6a,b). Alkyl halide was treated with compounds $(1 \mathrm{a}, \mathrm{b})$ in presence acetone to give of [-2-(methylthio)quinazoline-4(3H)-one] (7a,b), and with arylhalide in DMF gave the compounds [-2-((4-nitrophenyl)thio)quinazoline$4(3 \mathrm{H})$-one $)](8 \mathrm{a}, \mathrm{b})$. The structures of all prepared compounds have been elucidated using FTIR, ${ }^{1}$ HNMR, and MASS spectroscopy. The antibacterial activity was evaluated for $(1,5,6,7) \mathrm{a}-\mathrm{b}$ derivatives.
\end{abstract}

Keywords:2-mercaptoquinazoline-4(3H)-one, antibacterial activity.

\section{Introduction}

Quinazolinone is one of the leading and flourishing structures in medicinal chemistry [1]. Quinazolinone derivatives display a wide range of biological and pharmacological activities such as anticonvulsant, antiinflammatory, antitumor, analgesic, anticancer, cytotoxic, anticoccidial, antibacterial and antifungal [2-5]. Quinazolinone is a frequently encountered unit in natural products such as L-vasicinone [6], chrysogine [7,8] and drugs as methaqualone [9]. A new series of 2substitutedmercapto-quinazolin-4-one analogs was synthesized and screened. In the present study, the quinazolinone analogs were designed to contain a 2-substituted-thio functional group, this thioether moiety believed to bound to an electron-deficient carbon atom which identified as a possible pharmacophore of the antimicrobial activity [10]. On the other hand, hydrazides, hydrazones, or azomethines are of wide interest because of their diverse synthetic, biological, and clinical applications [11-12]. herein, We reported the synthesis of new hydrazone derivatives of S-linked substituted acetohydrazide, hydrazinyl, thioquinazolin, quinazolines with a number of substituted benzaldehyde in an attempt to obtain compounds with enhanced bioactivities [13].The new synthesized compounds were screened against gram-positive bacteria (E.coli and S.aureus) and gram-negative bacteria (K. pneumoniae, P.eruginose).

\section{Experimental:}

\section{Materials and Methods}

1. Melting point were recorded with Stuart melting point (Smp30) apparatus and were uncorrected.

2. Spectra date of (FT-IR) was recorded on Shimadzu FT-IR8400S spectrophotometer without $\mathrm{KBr}$ in chemistry department, college of Science, Mustansiriya University.

3. Mass spectra were recorded on a Shimadzu GC-MS Qp-2010 Ultra using (NCI-MS) mode negative chemical ionization in Chemistry Department, College of Science, Mustansiriya University.

4. ${ }^{1} \mathrm{H}-\mathrm{NMR}$ spectra were recorded on a BRUKER (300.13) MHZ spectrometer in 
DMSO-d6 as a solvent with TMS as an internal standard in Al-Albait university Jordan.

5. Thin layer Chromatography (TLC) was carried out by using alumina plates percoated with silica-gel, supplied by Merk. The compounds were detected with a 254-366nm UV Lamp.

6. The bacteria used were Escherichia coli, Klebsiella pneumoniae, pseudomonas eruginosa and Staphylococcus aureus in Chemistry department, college of Science, Mustansiriya University.

\section{1-Synthesis of substituted 3-(3-} chlorophenyl) -2-mercapto quinazolin4(3H)-one (1a,b) [14]

A mixture of substituted anthranilic acid (0.04 mole) and 3-chlorophenylisothiocyanate $(8.12 \mathrm{~g}, 0.04 \mathrm{~mole})$ in ethanol $(50 \mathrm{ml})$ was heated under reflux for $6 \mathrm{hr}$. The reaction mixture was cooled and solvent was evaporated under reduced pressure. The obtained residue was washed with petroleum ether, filtered, dried to give $(1 \mathrm{a}, \mathrm{b})$.

2.2-Synthesis of compounds ethyl 2-((3-)(3chlorophenyl)-4-oxo-3,4-dihydroquinazolin2-yl) thio) acetate and-6-hydroxy-4-oxo3,4dihydroquinazolin-2-yl) thio ) acetate $(2 a, b)[15]$.

To a solution of compounds $(1 \mathrm{a}, \mathrm{b})(0.02$ mole) and ethylchloroacetate $(2.45 \mathrm{~g}, 0.02$ mole $)$ in $(50 \mathrm{ml})$ absolute ethanol, fused $\mathrm{K}_{2} \mathrm{CO}_{3}$ (2.76 g, 0.02 mole) was added. The whole mixture was refluxed for $10 \mathrm{hr}$. The reaction mixture was filtered while hot and the filtrate was poured into ice cold water. The precipitated solid was filtered off, washed with water, dried and recrystallized from ethanol to give compounds $(2 \mathrm{a}, \mathrm{b})$.

2.3-Synthesis of compounds 2-((3-)(3chlorophenyl)-4-oxo-3,4- dihydroquinazolin2-yl) thio) acetohydrazide and 2 -hydrazinyl quinazolin -4(3H)-one (3-4) a,b [15].

A mixture of compounds (1-2) a,b (8 mmole) and hydrazin hydrate $(80 \%)(0.6 \mathrm{~g}$, $1.2 \mathrm{mmole})$ in $(50 \mathrm{ml})$ absolute ethanol was heated under reflux for $8 \mathrm{hr}$. The reaction mixture was cooled and solvent was evaporated under reduced pressure. The obtained residue was filtered, dried and recrystallized from ethyl acetate to give compounds (3-4) a,b.

2.4-Synthesis of compounds 2-((3-)(3chlorophenyl)-4-oxo-3,4-di hydro quinzolin2-yl) thio)- $\mathrm{N}^{-}$-(3-nitro benzylidene ) aceto hydrazide (5a,b), (E) -3- (3-chlorophenyl) 2- (2- (3- nitro benzylidene ) hydrazinyl) $(6 \mathrm{a}, \mathrm{b})[16]$.

A solution of hydrazide derivatives (3-4)a,b (2 mmole) in absolute ethanol $(30 \mathrm{ml})$ and 3-nitrobenzaldehyde ( $0.3 \mathrm{~g}, 2$ mmole) with (3 drops) of glacial acetic acid was refluxed for $8 \mathrm{hr}$. The reaction mixture was then cooled and solvent was evaporated under reduced pressure. The precipitate formed was poured into ice water, dried and recrystallized from ethanol to give compounds (5-6 a,b).

2.5-Synthesis of compounds 3-(3-(3chlorophenyl)-2-(methyl thio) quinazoline $(7 a, b)[14]$.

To a mixture of compounds (1a,b) (0.01mole) and anhydrous potassium carbonate (1.38 g, 0.01 mole) in dry acetone $(50 \mathrm{ml})$, methyl iodide ( $1.42 \mathrm{~g}, 0.01$ mole) was added dropwise. The reaction mixture was stirred and heated under reflux for $10 \mathrm{hr}$, the reaction mixture was filtered while hot and filtrate was concentrated under reduced pressure. The obtained crude product was recrystallized from petroleum ether to give compounds $(7 \mathrm{a}, \mathrm{b})$.

2.6-Synthesis of compounds 3-(3chlorophenyl)-2-((4-nitrophenyl) thio) quinazoline (8a, b) [14].

A mixture of compound (1a-b) (0.01 mole), 1-chloro- 4- nitro benzene ( $1.58 \mathrm{~g}, 0.01 \mathrm{~mole})$ and anhydrous potassium carbonate $(1.38 \mathrm{~g}$, $0.01 \mathrm{~mole})$ in DMF $(30 \mathrm{ml})$, was heated under reflux for $10 \mathrm{hr}$. The reaction mixture was filtered while hot and the filtrate was concentrated under reduced pressure. The obtained crude product was washed with cold water and recrystallized from ethanol to give compounds $(8 \mathrm{a}, \mathrm{b})$. 
Table (1)

The physical properties of compounds (1-8) a,b.

\begin{tabular}{|c|c|c|c|c|c|c|}
\hline Comp. No. & $-R$ & $M . F$ & M.wt & M.P/ ${ }^{\circ} \mathrm{C}$ & Yield \% & Colour \\
\hline $1 \mathrm{a}$ & $-\mathrm{H}$ & $\mathrm{C}_{14} \mathrm{H}_{9} \mathrm{~N}_{2} \mathrm{OSCl}$ & 288.5 & $302-304$ & 83 & White \\
\hline $1 \mathrm{~b}$ & $5-\mathrm{OH}$ & $\mathrm{C}_{14} \mathrm{H}_{9} \mathrm{~N}_{2} \mathrm{O}_{2} \mathrm{SCl}$ & 304.5 & 290 & 73 & White \\
\hline $2 \mathrm{a}$ & $-\mathrm{H}$ & $\mathrm{C}_{18} \mathrm{H}_{15} \mathrm{~N}_{2} \mathrm{O}_{3} \mathrm{SCl}$ & 374.5 & 136-139 & 67 & White \\
\hline $2 b$ & $5-\mathrm{OH}$ & $\mathrm{C}_{18} \mathrm{H}_{15} \mathrm{~N}_{2} \mathrm{O}_{4} \mathrm{SCl}$ & 390.5 & $161-163$ & 64 & White \\
\hline $3 a$ & $-\mathrm{H}$ & $\mathrm{C}_{16} \mathrm{H}_{13} \mathrm{~N}_{4} \mathrm{O}_{2} \mathrm{SCl}$ & 360.5 & $228-230$ & 60 & Green \\
\hline $3 \mathrm{~b}$ & $\overline{5-\mathrm{OH}}$ & $\mathrm{C}_{16} \mathrm{H}_{13} \mathrm{~N}_{4} \mathrm{O}_{3} \mathrm{SCl}$ & 376.5 & 237 & $\overline{c 58}$ & Green \\
\hline$\overline{4 \mathrm{aa}}$ & $2-\mathrm{H}$ & $\mathrm{C}^{\mathrm{\textrm {C } _ { 1 4 }} \mathrm{H}_{11} \mathrm{~N}_{4} \mathrm{OCl}}$ & 286.5 & $\overline{190-192}$ & $\overline{711}$ & White \\
\hline $4 \mathrm{bb}$ & $\overline{5-\mathrm{OH}}$ & $\mathrm{C}_{14} \mathrm{H}_{11} \mathrm{~N}_{4} \mathrm{O}_{2} \mathrm{Cl}$ & 302.5 & $215-217$ & 68 & White \\
\hline $5 \mathrm{a}$ & $-\mathrm{H}$ & $\mathrm{C}_{23} \mathrm{H}_{16} \mathrm{~N}_{5} \mathrm{O}_{4} \mathrm{SCl}$ & 493.5 & 282 & 56 & Yellow \\
\hline $5 \mathrm{~b}$ & $5-\mathrm{OH}$ & $\mathrm{C}_{23} \mathrm{H}_{16} \mathrm{~N}_{5} \mathrm{O}_{5} \mathrm{SCl}$ & 509.5 & 295-297 & 52 & Yellow \\
\hline $6 \mathrm{a}$ & $-\mathrm{H}$ & $\mathrm{C}_{21} \mathrm{H}_{14} \mathrm{~N}_{5} \mathrm{O}_{3} \mathrm{Cl}$ & 419.5 & $253-255$ & 57 & Green \\
\hline $6 \mathrm{~b}$ & $5-\mathrm{OH}$ & $\mathrm{C}_{21} \mathrm{H}_{14} \mathrm{~N}_{5} \mathrm{O}_{4} \mathrm{Cl}$ & 435.5 & $273-276$ & 54 & Green \\
\hline$\overline{7 \mathrm{a}}$ & $\begin{array}{l}-\mathrm{H} \\
\end{array}$ & $\mathrm{C}_{15} \mathrm{H}_{11} \mathrm{~N}_{2} \mathrm{OSCl}$ & 302.5 & 180-182 & $\overline{773}$ & White \\
\hline$\overline{7 b}$ & $\overline{5-\mathrm{OH}}$ & $\overline{\mathrm{C}_{15} \mathrm{H}_{11} \mathrm{~N}_{2} \mathrm{O}_{2} \mathrm{SCl}}$ & 318.5 & 195-197 & $\overline{669}$ & Yellow \\
\hline $8 \mathrm{a}$ & (-H & $\mathrm{C}_{20} \mathrm{H}_{12} \mathrm{~N}_{3} \mathrm{O}_{3} \mathrm{SCl}$ & 409.5 & $158-161$ & 72 & Yellow \\
\hline $8 \mathrm{~b}$ & 5-OH & $\mathrm{C}_{20} \mathrm{H}_{12} \mathrm{~N}_{3} \mathrm{O}_{4} \mathrm{SCl}$ & 425.5 & 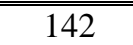 & $\overline{770}$ & Yellow \\
\hline
\end{tabular}

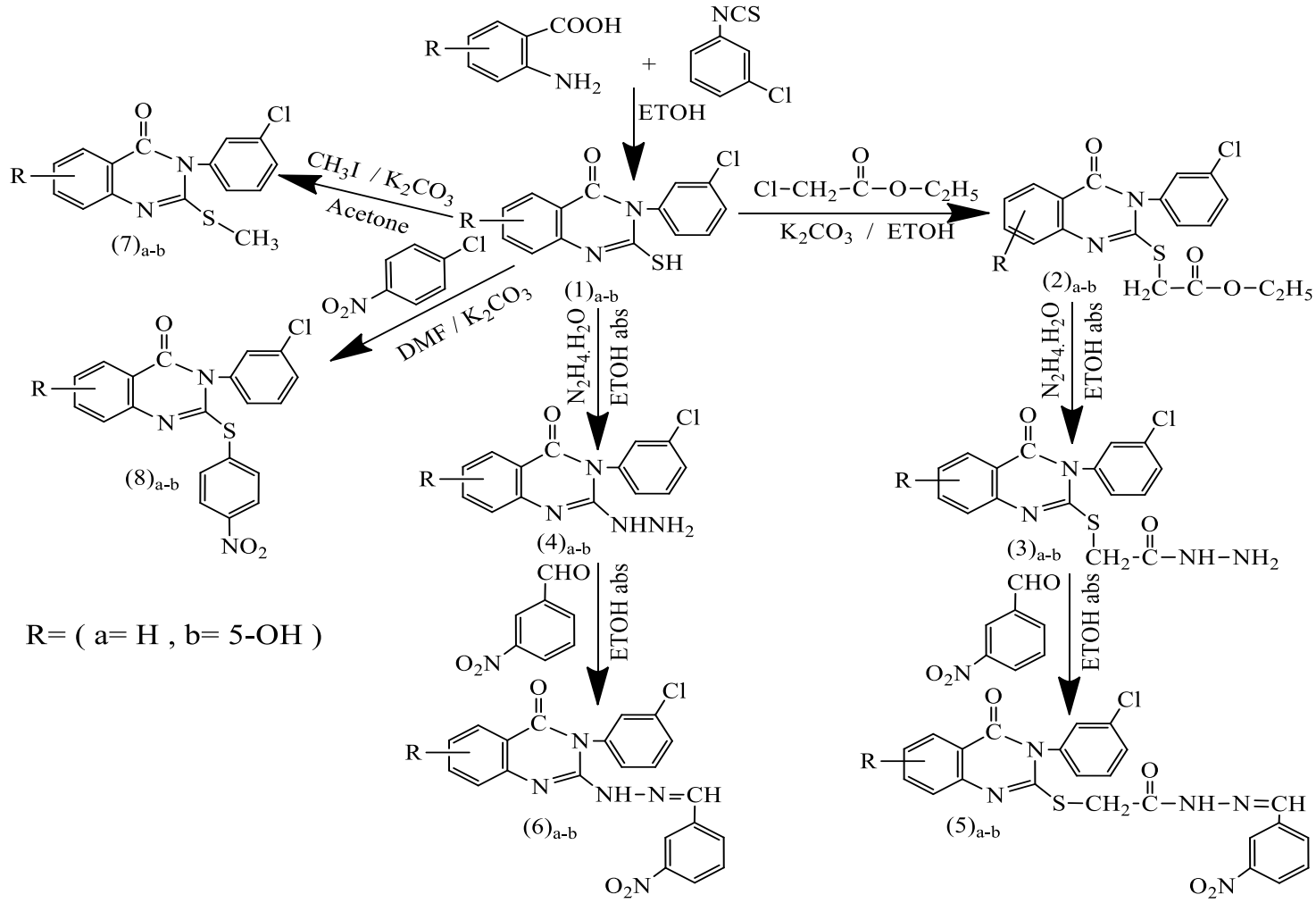

\section{Results and Discussion}

In the reaction of substituted anthranilic acid with 3-chloro phenyl isothiocyanate in ethanol [14]. The structural assignment of the product was based on its melting points and spectral data (FT-IR, ${ }^{1} \mathrm{H}-\mathrm{NMR}$ and MASS) $[17,18]$. The FT-IR spectrum of compounds (1a), Fig.(1) and (2a), Fig.(2) showed at $\left(3271 \mathrm{~cm}^{-1}\right)$ for $(\mathrm{N}-\mathrm{H})$ group band, at $(2250$ $\left.2450 \mathrm{~cm}^{-1}\right)$ for $(\mathrm{S}-\mathrm{H})$ group band, at (1656-

\section{Scheme (1)}

$\left.1681 \mathrm{~cm}^{-1}\right)$ for $(\mathrm{C}=\mathrm{O})$ group band and at $(1643$ $\left.1649 \mathrm{~cm}^{-1}\right)$ due to cyclic $(\mathrm{C}=\mathrm{N})$ stretching is also observed. ${ }^{1} \mathrm{H}-\mathrm{NMR}$ spectrum of compound (1a), Fig.(3) shows the following characteristic chemical shifts in (DMSO-d 6 ) at $13.1 \mathrm{ppm}(\mathrm{s}, 1 \mathrm{H}, \mathrm{NH}-\mathrm{SH})$, at 7.34-7.99 ppm (m,8H, Ar-H). Compound (1b), Fig.(4) shows at $12.92 \mathrm{ppm}(\mathrm{s}, 1 \mathrm{H}, \mathrm{NH}-\mathrm{SH})$, at $9.95 \mathrm{ppm}$ $(\mathrm{s}, 1 \mathrm{H}, \mathrm{OH})$, at 7.29-7.95 ppm $(\mathrm{m}, 7 \mathrm{H}, \mathrm{Ar}-\mathrm{H})$. The MS spectrum of compound (1a), Fig.(5) 
shows the molecular ion peak at $\mathrm{M} / \mathrm{Z}=288$ and (1b), Fig.(6), the molecular ion peak at $\mathrm{M} / \mathrm{Z}=304$. The quinazolinones (1a-b) were converted to corresponding esters $(2 \mathrm{a}-\mathrm{b})$ by treating with ethyl chloroacetate in ethanol using acatalytic amount of anhydrous potassium carbonate [15]. The FT-IR spectrum of compounds $(2 \mathrm{a}, \mathrm{b})$, shows the disappearance of (NH-SH) absorption and appearance of ester carbonyl stretching band at $\left(1735 \mathrm{~cm}^{-1}\right)$, (C-H) aliphatic stretching band at (2929$\left.2989 \mathrm{~cm}^{-1}\right),(\mathrm{C}-\mathrm{H})$ aliphatic bending band at $\left(1363-1484 \mathrm{~cm}^{-1}\right)$ and at $\left(1232-1301 \mathrm{~cm}^{-1}\right)$ belongs to asymmetric and symmetric $(\mathrm{C}-\mathrm{O}-\mathrm{C})$ group. ${ }^{1} \mathrm{H}-\mathrm{NMR}$ spectrum of compound (2a), Fig.(7) shows the appearance $\left(\mathrm{t}, 3 \mathrm{H}, \mathrm{CH}_{2} \mathrm{CH} 3\right)$ at $1.24 \mathrm{ppm}$, at $4 \mathrm{ppm}\left(\mathrm{s}, 2 \mathrm{H}, \mathrm{S}-\mathrm{CH}_{2}\right)$, at $4.18 \mathrm{ppm}\left(\mathrm{q}, 2 \mathrm{H}, \mathrm{OCH}_{2} \mathrm{CH}_{3}\right)$ and at $7.3-8 \mathrm{ppm}$ $(\mathrm{s}, 8 \mathrm{H}, \mathrm{Ar}-\mathrm{H})$. The FT-IR spectrum of compounds $(3 a, b)$ shows the appearance of primary $\left(\mathrm{NH}_{2}\right)$, along with a shoulder band at (3184-3321 cm $\left.\mathrm{cm}^{-1}\right)$, secondary $(\mathrm{N}-\mathrm{H})$ band at (3105-3217 $\left.\mathrm{cm}^{-1}\right)$, besides the disappearance of band at $\left(1735 \mathrm{~cm}^{1}\right)$ due to carbonyl group of ester and appearance of band at (1643-1651 $\left.\mathrm{cm}^{-1}\right)$ attributed for carbonyl group of hydrazide. ${ }^{1} \mathrm{H}-\mathrm{NMR}$ spectrum of compound (3a), Fig.(8) shows the appearance of $(\mathrm{s}, 1 \mathrm{H}$, $\mathrm{N}-\mathrm{H})$ peak at $9.5 \mathrm{ppm}$, at $5.69 \mathrm{ppm}$ $\left(\mathrm{s}, 2 \mathrm{H}, \mathrm{NH}_{2}\right)$, at $4.36 \mathrm{ppm}\left(\mathrm{s}, 2 \mathrm{H}, \mathrm{S}-\mathrm{CH}_{2}\right)$ and at 7-8 ppm $(\mathrm{m}, 8 \mathrm{H}, \mathrm{Ar}-\mathrm{H})$. The FT-IR spectral of compounds (4a-b), show the appearance of primary $\left(\mathrm{NH}_{2}\right)$, along with a shoulder band at $\left(3291-3342 \mathrm{~cm}^{-1}\right)$, secondary $(\mathrm{N}-\mathrm{H})$ band at (3186-3219 $\mathrm{cm}^{-1}$ ), besides the disappearance of band at (2250-2450 $\left.\mathrm{cm}^{-1}\right)$ attributed to (S-H) group. ${ }^{1} \mathrm{H}-\mathrm{NMR}$ spectrum of compound (4a) Fig.(9) shows the appearance of $\left(\mathrm{s}, 2 \mathrm{H}, \mathrm{NH}_{2}\right)$ at $5.71 \mathrm{ppm}$, at $9.54 \mathrm{ppm}(\mathrm{s}, 1 \mathrm{H}, \mathrm{NH})$ and at $7.25-8 \mathrm{ppm}(\mathrm{m}, 8 \mathrm{H}, \mathrm{Ar}-\mathrm{H})$, compound (4b) Fig.(10) shows the appearance of $(\mathrm{s}, 1 \mathrm{H}, \mathrm{NH})$ peak at $9.65 \mathrm{ppm}$, at $9.35 \mathrm{ppm}$ $(\mathrm{s}, 1 \mathrm{H}, \mathrm{OH})$, at $5.67 \mathrm{ppm}\left(\mathrm{s}, 2 \mathrm{H}, \mathrm{NH}_{2}\right)$ and at 7.14-8ppm (s,7H,Ar-H). The prepared compounds (5-6) a,b were synthesized by the reaction of compound (3-4) a,b and 3nitrobenzaldehyde in ethanol in presence of acataylic amount of glacial acetic acid [16]. The FT-IR spectrum of compounds (5-6)a,b, shows the disappearance of $\left(\mathrm{NH}_{2}\right)$ stretching of hydrazine group and appearance of band for azomethine $(\mathrm{C}=\mathrm{N})$ group at $\left(1608-1620 \mathrm{~cm}^{-1}\right)$.
${ }^{1} \mathrm{H}-\mathrm{NMR}$ (DMSO-d6) spectrum of compound (6a), Fig.(11) shows the appearance of $(\mathrm{s}, 1 \mathrm{H}, \mathrm{NH})$ peak at $10.85 \mathrm{ppm}$, at $8.75 \mathrm{ppm}$ $(\mathrm{s}, 1 \mathrm{H}, \mathrm{N}=\mathrm{CH}) \quad$ and $\mathrm{at} 7.18-8.38 \quad \mathrm{ppm}$ $(\mathrm{m}, 12 \mathrm{H}, \mathrm{Ar}-\mathrm{H})$. The MS spectrum of compound (6b), Fig.(12) shows the molecular ion peak at $M / z=433$. The FT-IR spectrum of compounds (7-8) a,b, shows the appearance band at (1014-1066 $\left.\mathrm{cm}^{-1}\right)$ for $(\mathrm{C}-\mathrm{S}),(\mathrm{C}-\mathrm{H})$ aliphatic band at $\left(2972-2985 \mathrm{~cm}^{-1}\right)$, besides the disappearance of band at $\left(2250-2450 \mathrm{~cm}^{-1}\right)$ due to (S-H) group. ${ }^{1} \mathrm{H}-\mathrm{NMR}$ (DMSO - d6) spectrum of compound (7a), Fig.(13) shows the appearance of $\left(\mathrm{s}, 1 \mathrm{H}, \mathrm{S}-\mathrm{CH}_{3}\right)$ at $3.35 \mathrm{ppm}$, at $6.75-8 \mathrm{ppm}(\mathrm{s}, 8 \mathrm{H}, \mathrm{Ar}-\mathrm{H})$, compound $(7 \mathrm{~b})$ Fig.(14) shows the appearance of (s,12H,Ar-H) at 7.45-8 ppm, compound (8b) Fig.(15) Shows the appearance of $(\mathrm{s}, 1 \mathrm{H}, \mathrm{OH})$ at $10 \mathrm{ppm}$, at 7.26-8 ppm (s,12H,Ar-H). The MS spectrum of compound (7a) Fig.(16) illustrates $\mathrm{M} / \mathrm{Z}=302,[\mathrm{M}+1]^{+}=303$ and $[\mathrm{M}+29]^{+}=333$ due to using another ionization mode chemical ionization(CI). All the spectral data for other compounds are listed in Table (2). 
Table (2)

FT-IR spectral data of compounds (1-8) a,b.

\begin{tabular}{|c|c|c|c|c|c|c|}
\hline $\begin{array}{l}\text { Comp } \\
\text { No. }\end{array}$ & $\begin{array}{l}v(\mathrm{C}-\mathrm{H}) \\
\text { aromatic } \\
\mathrm{cm}^{-1}\end{array}$ & $\begin{array}{l}v(C=C) \\
\text { aromatic } \\
\mathrm{cm}^{-1}\end{array}$ & $\begin{array}{c}v(C=O) \\
\text { quinazoline } \\
\mathrm{cm}^{-1}\end{array}$ & $\begin{array}{c}v(C=N) \\
\text { endo } \mathrm{cm}^{-1}\end{array}$ & $\begin{array}{c}v(C-N) \\
c m^{-1}\end{array}$ & Other bands $\mathrm{cm}^{-1}$ \\
\hline $1 \mathrm{a}$ & 3095 & 1616 & 1681 & 1643 & 1087 & $\begin{array}{l}v(\mathrm{~N}-\mathrm{H}) 3271 \quad v(\mathrm{C}-\mathrm{S}) 1016 \\
v(\mathrm{C}-\mathrm{Cl}) 758 v(\mathrm{C}-\mathrm{SH}) 2250\end{array}$ \\
\hline $1 b$ & 3047 & 1602 & 1656 & 1649 & 1082 & $\begin{array}{l}v(\mathrm{C}-\mathrm{OH}) 3244 \text { v(C-O) } 1234 \quad v(\mathrm{C}-\mathrm{S}) 1018 \\
v(\mathrm{C}-\mathrm{S}) 1018 v(\mathrm{C}-\mathrm{Cl}) 732 \quad v(\mathrm{C}-\mathrm{SH}) 2450\end{array}$ \\
\hline $2 \mathrm{a}$ & 3095 & 1606 & 1695 & 1653 & 1091 & $\begin{array}{c}v(\mathrm{C}-\mathrm{S}) 1016 v(\mathrm{C}=\mathrm{O}) \text { ester } 1735 \quad v(\mathrm{C}-\mathrm{O}) 1301 \\
v(\mathrm{C}-\mathrm{H}) \text { Aliphatic } 2929-2987 \\
v(\mathrm{C}-\mathrm{Cl}) 797 \\
v(\mathrm{C}-\mathrm{H}) \text { Bending } 1375-1467\end{array}$ \\
\hline $2 b$ & 3084 & 1616 & 1672 & 1654 & 1089 & $\begin{array}{c}v(\mathrm{C}-\mathrm{OH}) 3419 \text { v }(\mathrm{C}=\mathrm{O}) \text { ester } 1735 v(\mathrm{C}-\mathrm{O}) 1232 \\
v(\mathrm{C}-\mathrm{Cl}) 736 v(\mathrm{C}-\mathrm{H}) \text { Bending 1363-1484 } \\
v(\mathrm{C}-\mathrm{H}) \text { Aliphatic } 2929-2987 v(\mathrm{C}-\mathrm{S}) 1020\end{array}$ \\
\hline $3 \mathrm{a}$ & 3093 & 1610 & 1678 & 1639 & 1093 & $\begin{array}{c}v\left(\mathrm{~S}-\mathrm{CH}_{2}\right) 1475 \text { v(C-S) } 1006 \text { v }(\mathrm{C}-\mathrm{Cl}) 761 \\
v(\mathrm{C}-\mathrm{H}) \text { Aliphatic } 2955 v(\mathrm{C}=\mathrm{O}) \text { amide } 1651 \\
v\left(\mathrm{NH}_{2}\right) 3321-3296 v(\mathrm{~N}-\mathrm{H}) 3217\end{array}$ \\
\hline $3 b$ & 3016 & 1587 & 1662 & 1608 & 1089 & $\begin{array}{c}v(\mathrm{C}-\mathrm{OH}) 3410 v\left(\mathrm{~S}-\mathrm{CH}_{2}\right) 1485 v(\mathrm{C}-\mathrm{O}) 1217 \\
v(\mathrm{C}-\mathrm{S}) 1010 v(\mathrm{C}-\mathrm{Cl}) 750 v(\mathrm{C}=\mathrm{O}) \text { amide } 1643 \\
v(\mathrm{C}-\mathrm{H}) \text { Aliphatic } 2937 \quad v\left(\mathrm{NH}_{2}\right) 3302 \\
v(\mathrm{~N}-\mathrm{H}) 3105 \\
\end{array}$ \\
\hline $4 a$ & 3093 & 1606 & 1678 & 1643 & 1093 & $\begin{array}{l}v(\mathrm{C}-\mathrm{Cl}) 761 \text { v (N-H) } 1573 \text { bending } \\
v\left(\mathrm{NH}_{2}\right) 3291-3342 \quad v(\mathrm{~N}-\mathrm{H}) 3219\end{array}$ \\
\hline $4 b$ & 3051 & 1604 & 1664 & 1635 & 1085 & $\begin{array}{c}v(\mathrm{C}-\mathrm{OH}) 3435 v(\mathrm{C}-\mathrm{O}) 1236 \quad v(\mathrm{C}-\mathrm{Cl}) 748 \\
v(\mathrm{~N}-\mathrm{H}) 1585 \text { bending } v\left(\mathrm{NH}_{2}\right) 3273-3302 \\
v(\mathrm{~N}-\mathrm{H}) 3186\end{array}$ \\
\hline $\begin{array}{l}\text { Comp } \\
\text { No. }\end{array}$ & $\begin{array}{c}v(\mathrm{~N}-\mathrm{H}) \\
\mathrm{cm}^{-1}\end{array}$ & $\begin{array}{l}v(C=O) \\
\text { quinazoli } \\
n \mathrm{~cm}^{-1}\end{array}$ & $\begin{array}{l}v(C=O) \\
\text { amide } \mathrm{cm}^{-1}\end{array}$ & $\begin{array}{l}v(C=N) \\
\text { exo } \mathrm{cm}^{-1}\end{array}$ & $\begin{array}{c}(C=N) \\
\text { endo } \\
\mathrm{cm}^{-1}\end{array}$ & Other bonds $\mathrm{cm}^{-1}$ \\
\hline $5 \mathrm{a}$ & 3120 & 1675 & 1666 & 1620 & 1591 & $\begin{array}{c}v(\mathrm{C}=\mathrm{C}) 1563 \quad v(\mathrm{C}-\mathrm{N}) 1097 \\
v\left(\mathrm{C}-\mathrm{NO}_{2}\right) 1346-1543 \quad v(\mathrm{C}-\mathrm{Cl}) 748 \\
v(\mathrm{C}-\mathrm{H}) \text { aromatic } 3058 \quad v(\mathrm{C}-\mathrm{H}) \text { aliphatic } 2933\end{array}$ \\
\hline $5 b$ & 3152 & 1670 & 1650 & 1617 & 1585 & $\begin{array}{c}v(\mathrm{C}=\mathrm{C}) 1560 v(\mathrm{C}-\mathrm{N}) 1091 \quad v(\mathrm{C}-\mathrm{Cl}) 756 \\
v\left(\mathrm{C}-\mathrm{NO}_{2}\right) 1335-1548 \\
v(\mathrm{C}-\mathrm{H}) \text { aromatic } 3050 \quad v(\mathrm{C}-\mathrm{H}) \text { aliphatic } 2982 \\
v(\mathrm{C}-\mathrm{OH}) 3417\end{array}$ \\
\hline $6 a$ & 3129 & 1673 & - & 1610 & 1580 & $\begin{array}{c}v(\mathrm{C}=\mathrm{C}) 1568 \quad v(\mathrm{C}-\mathrm{N}) 1085 \\
v\left(\mathrm{C}-\mathrm{NO}_{2}\right) 1340-1548 \quad v(\mathrm{C}-\mathrm{Cl}) 765 \\
v(\mathrm{C}-\mathrm{H}) \text { aromatic } 3070 \quad v(\mathrm{C}-\mathrm{H}) \text { aliphatic } 2923\end{array}$ \\
\hline $6 b$ & 3172 & 1680 & - & 1608 & 1587 & $\begin{array}{c}v(\mathrm{C}=\mathrm{C}) 1561 \quad v(\mathrm{C}-\mathrm{N}) 1120 \\
v\left(\mathrm{C}-\mathrm{NO}_{2}\right) 1356-1534 \quad v(\mathrm{C}-\mathrm{Cl}) 771 \\
v(\mathrm{C}-\mathrm{H}) \text { aromatic3030 } \quad v(\mathrm{C}-\mathrm{H}) \text { aliphatic } 2912 \\
v(\mathrm{C}-\mathrm{OH}) 3395\end{array}$ \\
\hline $\begin{array}{l}\text { Comp } \\
\text { No. }\end{array}$ & $\begin{array}{c}\mathrm{v}(\mathrm{C}-\mathrm{H}) \\
\text { aromatic } \\
\mathrm{cm}^{-1}\end{array}$ & $\begin{array}{l}v(C=O) \\
\text { quinazoli } \\
\text { ncm }^{-1}\end{array}$ & $\begin{array}{l}v(C=N) \\
\text { endocm }^{-1}\end{array}$ & $\begin{array}{c}v(C-S) \\
c m^{-1}\end{array}$ & $\begin{array}{c}v(C-N) \\
c m^{-1}\end{array}$ & Other bands $\mathrm{cm}^{-1}$ \\
\hline $7 \mathrm{a}$ & 3088 & 1680 & 1643 & 1014 & 1089 & $\begin{array}{c}v(\mathrm{C}-\mathrm{H}) \text { aliphatic } 2985 \quad v(\mathrm{C}=\mathrm{C}) \text { aromatic } 1604 \\
v\left(\mathrm{~S}-\mathrm{CH}_{3}\right) \text { bending } 1429, \mathrm{v}(\mathrm{C}-\mathrm{Cl}) 767\end{array}$ \\
\hline $7 \mathrm{~b}$ & 3078 & 1681 & 1664 & 1018 & 1095 & $\begin{array}{c}v(\mathrm{C}-\mathrm{OH}) 3217 \quad v(\mathrm{C}-\mathrm{O}) 1243 \\
v(\mathrm{C}-\mathrm{H}) \text { aliphatic } 2972 \quad v(\mathrm{C}=\mathrm{C}) \text { aromatic } 1637 \\
v\left(\mathrm{~S}-\mathrm{CH}_{3}\right) \text { bending } 1454 \quad v(\mathrm{C}-\mathrm{Cl}) 760\end{array}$ \\
\hline $8 \mathrm{a}$ & 3076 & 1685 & 1640 & 1066 & 1112 & $\begin{array}{c}\mathrm{v}(\mathrm{C}=\mathrm{C}) \text { aromatic1579 } v\left(\mathrm{C}-\mathrm{NO}_{2}\right) 1381-1525 \mathrm{v}(\mathrm{C}- \\
\mathrm{Cl}) 748\end{array}$ \\
\hline $8 b$ & 3092 & 1687 & 1651 & 1027 & 1098 & $\begin{array}{ccc}v(\mathrm{C}-\mathrm{OH}) 3224 & v(\mathrm{C}-\mathrm{O}) 1248 \quad v(\mathrm{C}-\mathrm{Cl}) 757 \\
v(\mathrm{C}=\mathrm{C}) \text { aromatic } & 1618 \quad v\left(\mathrm{C}-\mathrm{NO}_{2}\right) 1383-1527\end{array}$ \\
\hline
\end{tabular}




\section{Microbiological Method}

The synthesized compounds $(1,5,6,7)$ a-b were screened in vitro for antimicrobial activity. The antibacterial test was performed according to the disc diffusion method [19]. The prepared agar and petridishes were sterilized by autoclaving for $(15 \mathrm{~min})$ at $121^{\circ} \mathrm{C}$, the agar plates were surface inoculated uniformly from the broth culture of the test microorganisms. In the solidified medium suitably spaced apart holes were made all $(6 \mathrm{~mm})$ in diameter. These holes were filled with $(100 \mu \mathrm{g} / \mathrm{ml})$ of the prepared compounds (1 $\mathrm{mg}$ of the compound dissolved in $1 \mathrm{ml}$ of DMSO solvent). These plates were incubated at $37{ }^{\circ} \mathrm{C}$ for $(24 \mathrm{hr})$. The inhibition zone caused by the various compounds were examined [20]. The results of the preliminary screening tests are listed in Table (3).

Table (3)

Antibacterial activities of some of the synthesized compounds $(1,5,6,7) a-b$.

\begin{tabular}{|c||c||c||c||c|}
\hline Comp. No & $E$. coli & K.pneumoniae & P. eruginosa & S.aureus \\
\hline \hline $1 \mathrm{a}$ & ++ & - & - & + \\
\hline \hline $1 \mathrm{~b}$ & +++ & - & - & ++ \\
\hline $5 \mathrm{a}$ & +++ & - & - & + \\
\hline \hline $5 \mathrm{~b}$ & +++ & - & - & ++ \\
\hline \hline $6 \mathrm{a}$ & ++ & - & - & ++ \\
\hline \hline $6 \mathrm{~b}$ & +++ & - & - & ++ \\
\hline \hline $7 \mathrm{a}$ & - & - & - & + \\
\hline \hline $7 \mathrm{~b}$ & + & - & - & + \\
\hline
\end{tabular}

Note: (-)=NO inhibition, $(+)=6-9 \mathrm{~mm}$ weak activity, $(++)=10-14 \mathrm{~mm}$ moderate activity, $(+++)=15-24 \mathrm{~mm}$ remarkable activity.

\section{Conclusion}

The screening results indicate that compounds (1b,5a,5b, and 6b) showed the remarkable activity against $E$-coli, compounds $(1 \mathrm{a}, 6 \mathrm{a})$ showed moderate activity on this bacteria, compound (7b) weak activity and compound (7a) no inhibition against $E$.coli. Compounds (1a,5a and 7b) weak activity against S.aureus and compound (7a) showed no inhibition against $S$. aureus. This means that $(1,5,6,7)$ a-b are inactive against $K$. pneumoniae and P.aeruginosa.

\section{Conclusion}

In conclusion, we describe procedure for the 2, 3-disusituted quinazoline derivatives were synthesized via reaction anthranilic acid derivatives and 3-chlorophenylisothiocyanate with ethanol obtained quinazoline- $4(3 \mathrm{H})$ one and reacted of aromatic aldehyde, aryl halide, aryl alkyl. The synthesized compounds were characterized by FT-IR, ${ }^{1} \mathrm{H}-\mathrm{NMR}$, MASS and antibacterial screening for the some compounds.

\section{Acknowledgments}

The authors would like to express their special thanks to the staff members of the department of chemical, collage of science, Mustansiriya University, Baghdad, Iraq, for performing the FT-IR, mass spectrophotometer and antibacterial testing of the some synthesis compounds. 


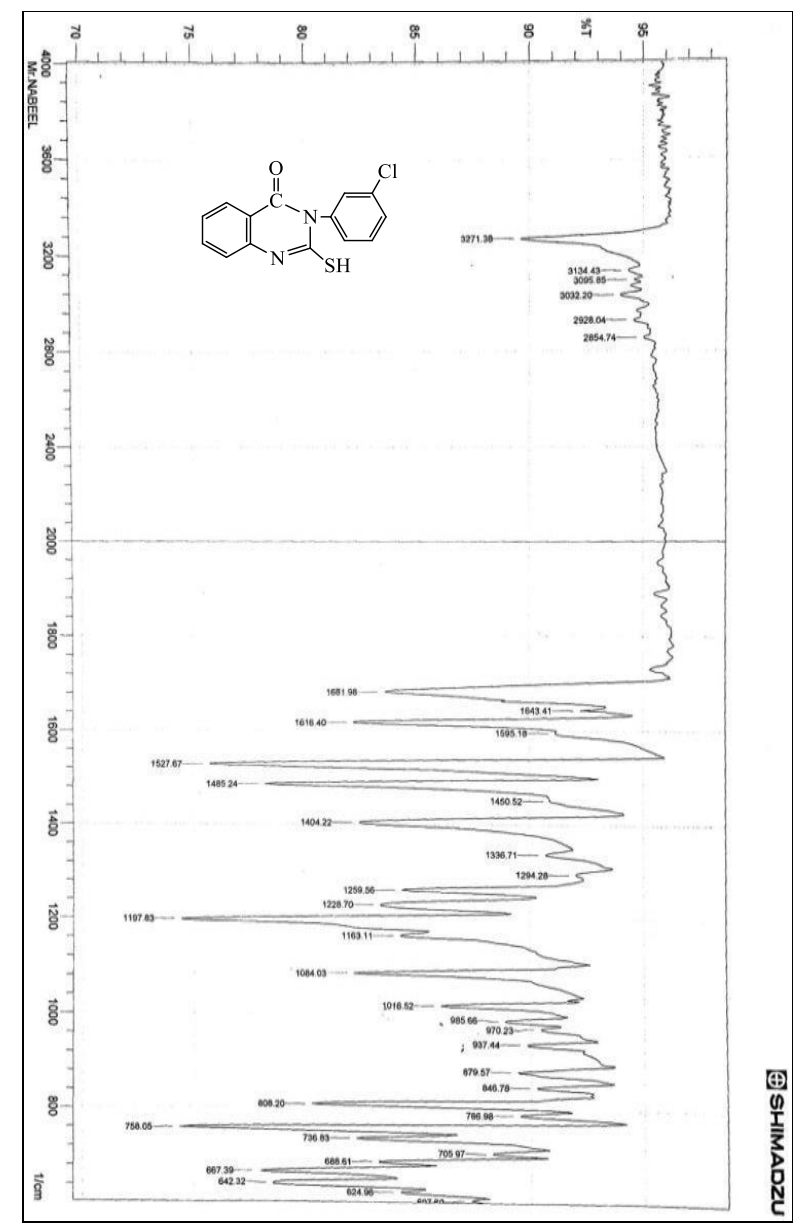

Fig.(1) FTIR spectum of compound (1a).

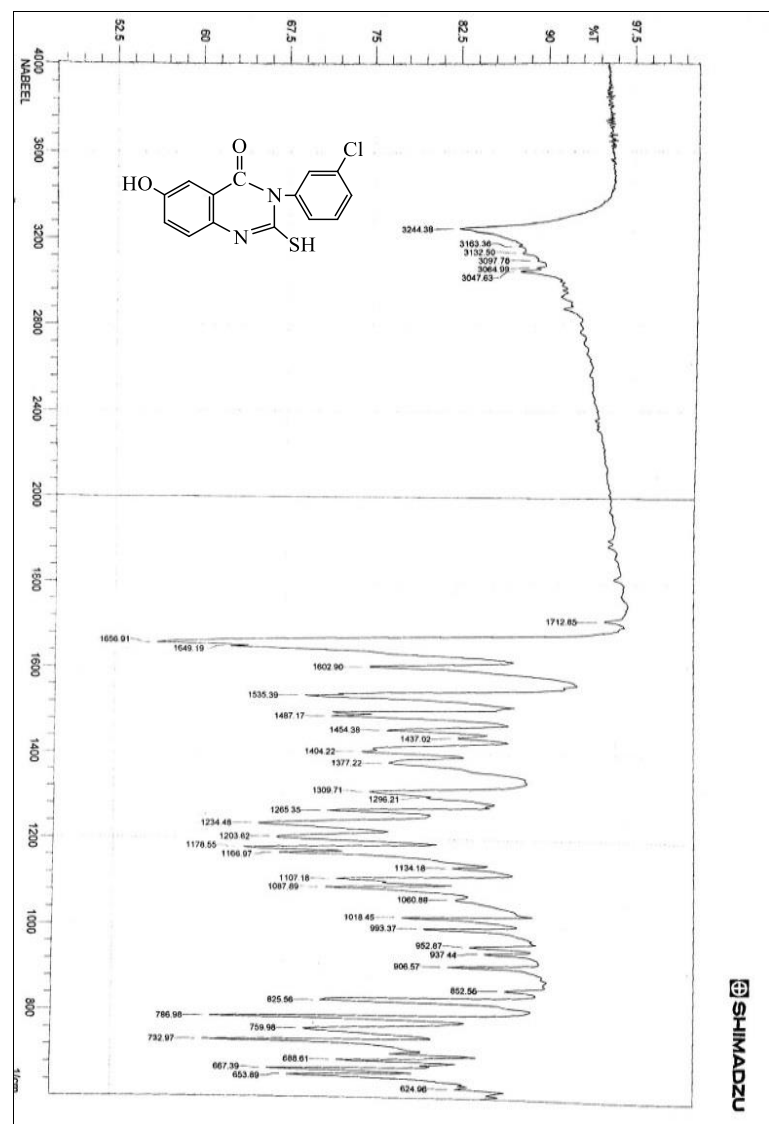

Fig.(2) FTIR spectrum of compound (1b).

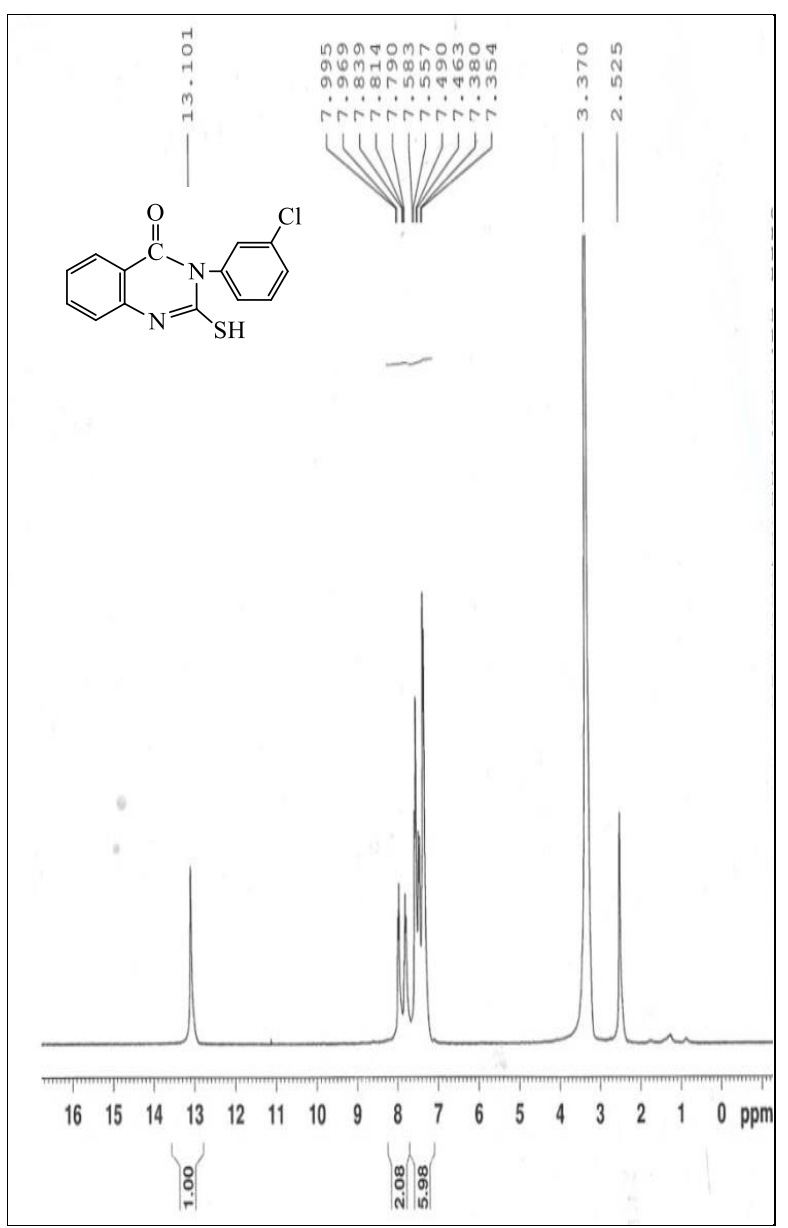

Fig.(3) ${ }^{I}$ H-NMR spectum of compound (1a).

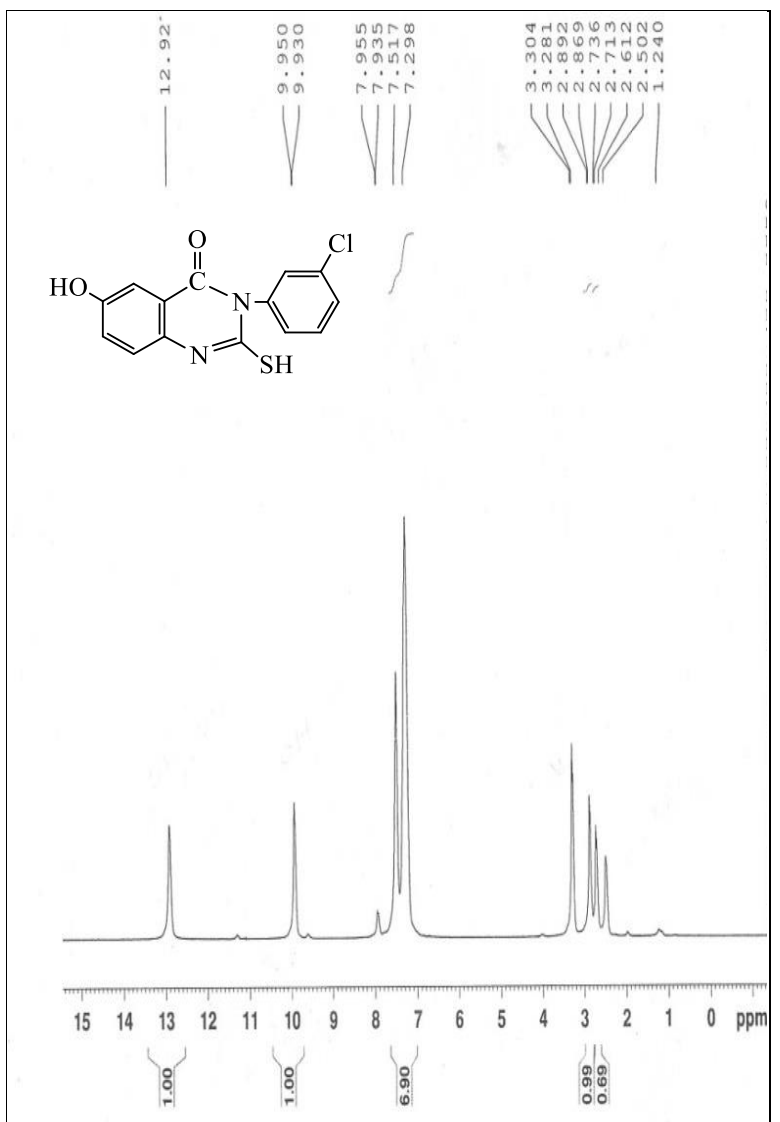

Fig.(4) ${ }^{I} \mathrm{H}$-NMR spectrum of compound (1b). 
Souad J. Lfta

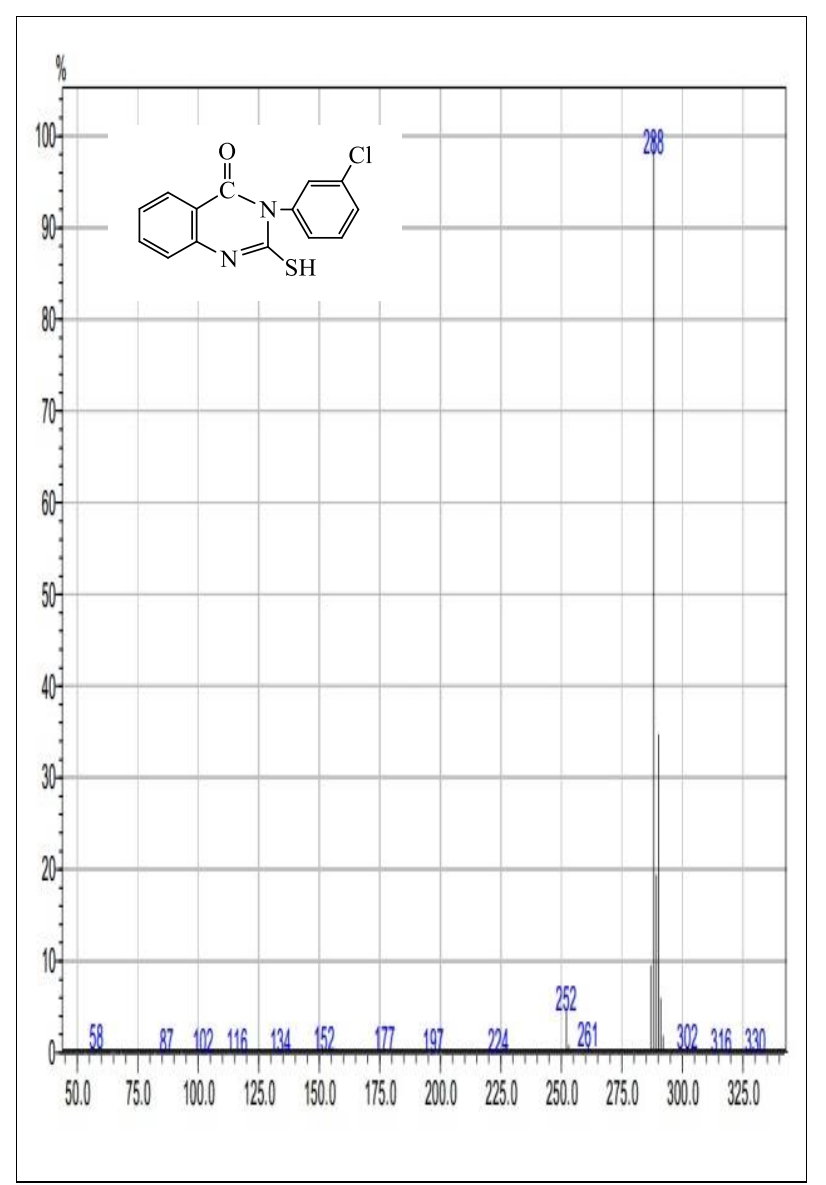

Fig.(5) MASS spectrum of compound (1a).

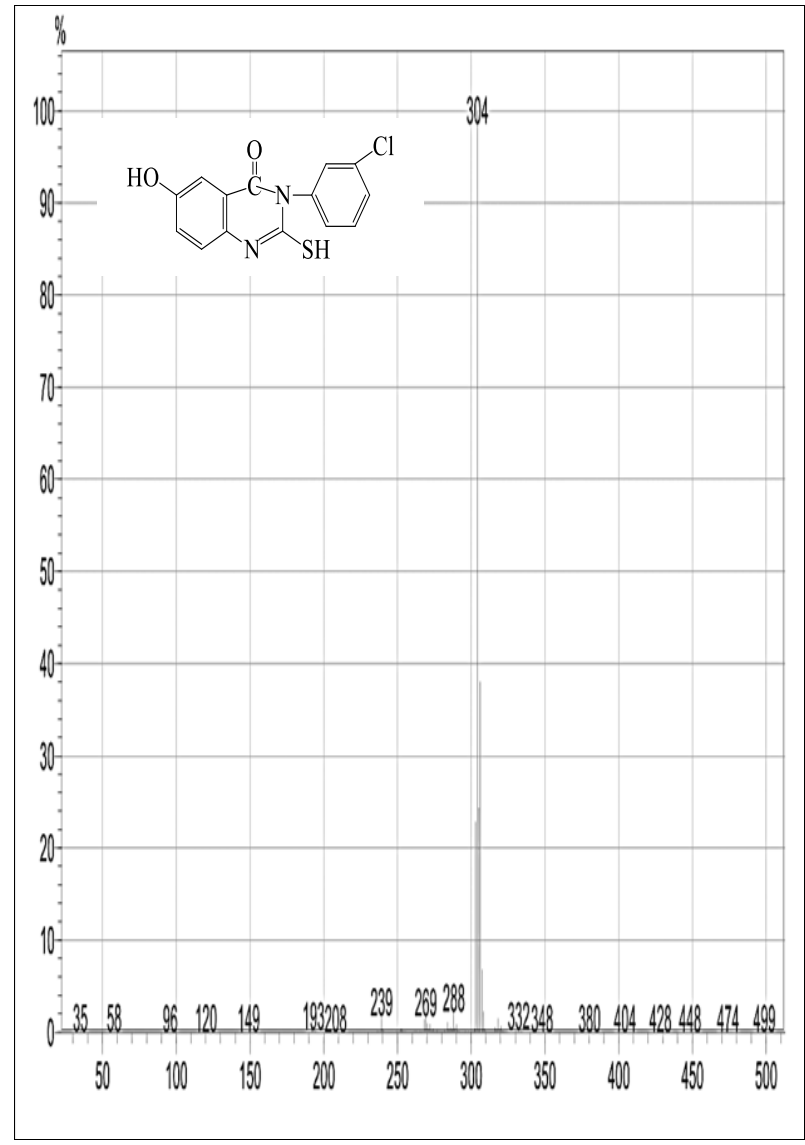

Fig.(6) MASS spectrum of compound (1b).

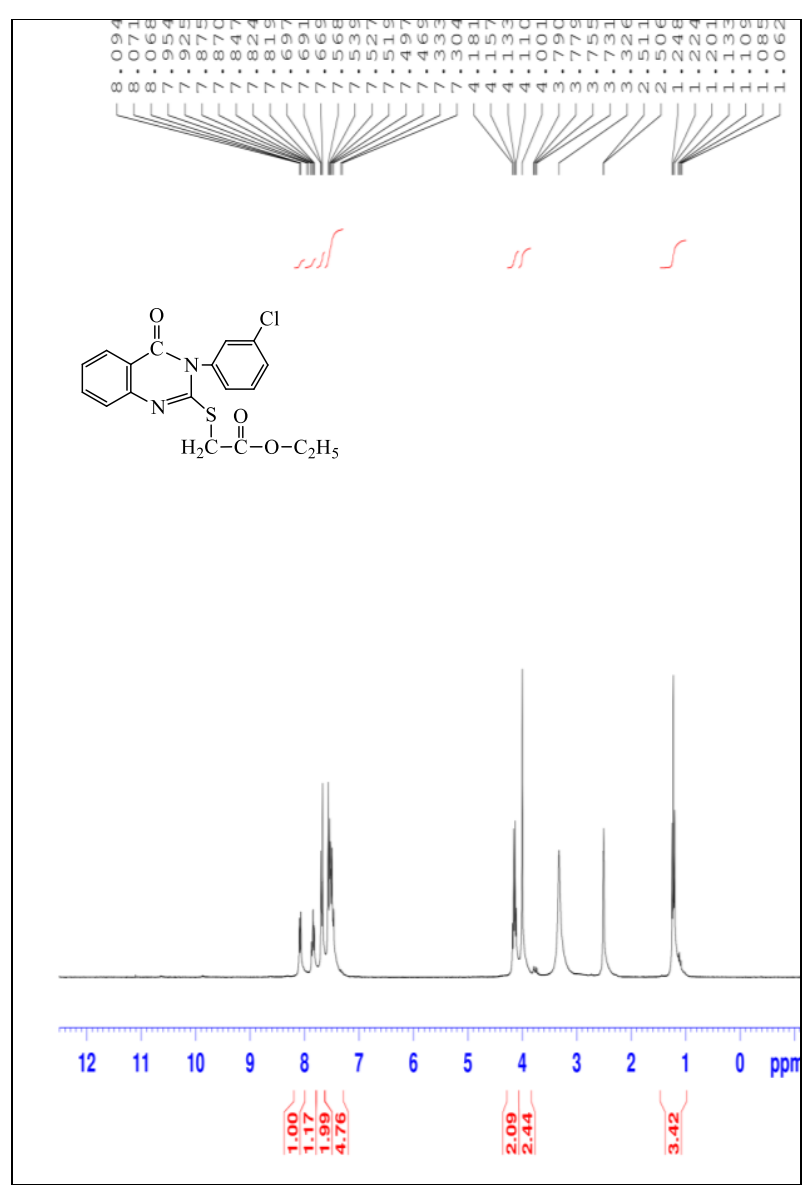

Fig.(7) ${ }^{1}$ H-NMR spectrum of compound (2a).

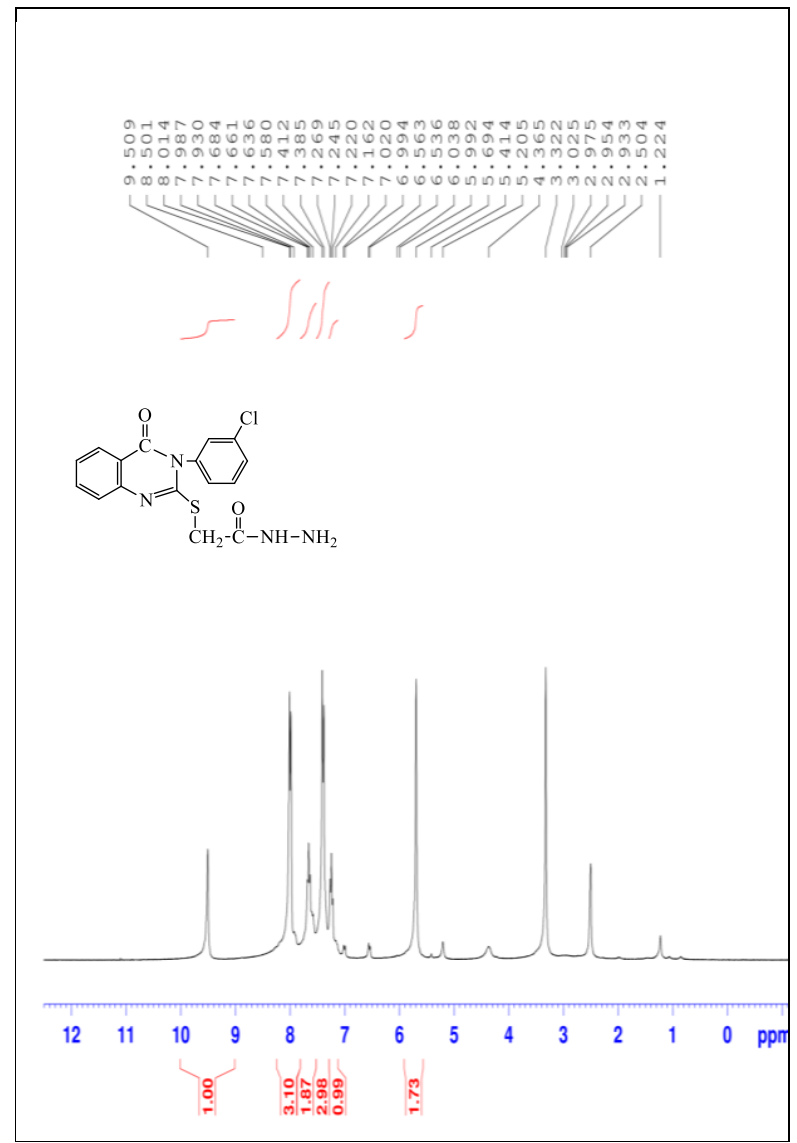

Fig.(8) ${ }^{I} \mathrm{H}$-NMR spectrum of compound (3a). 


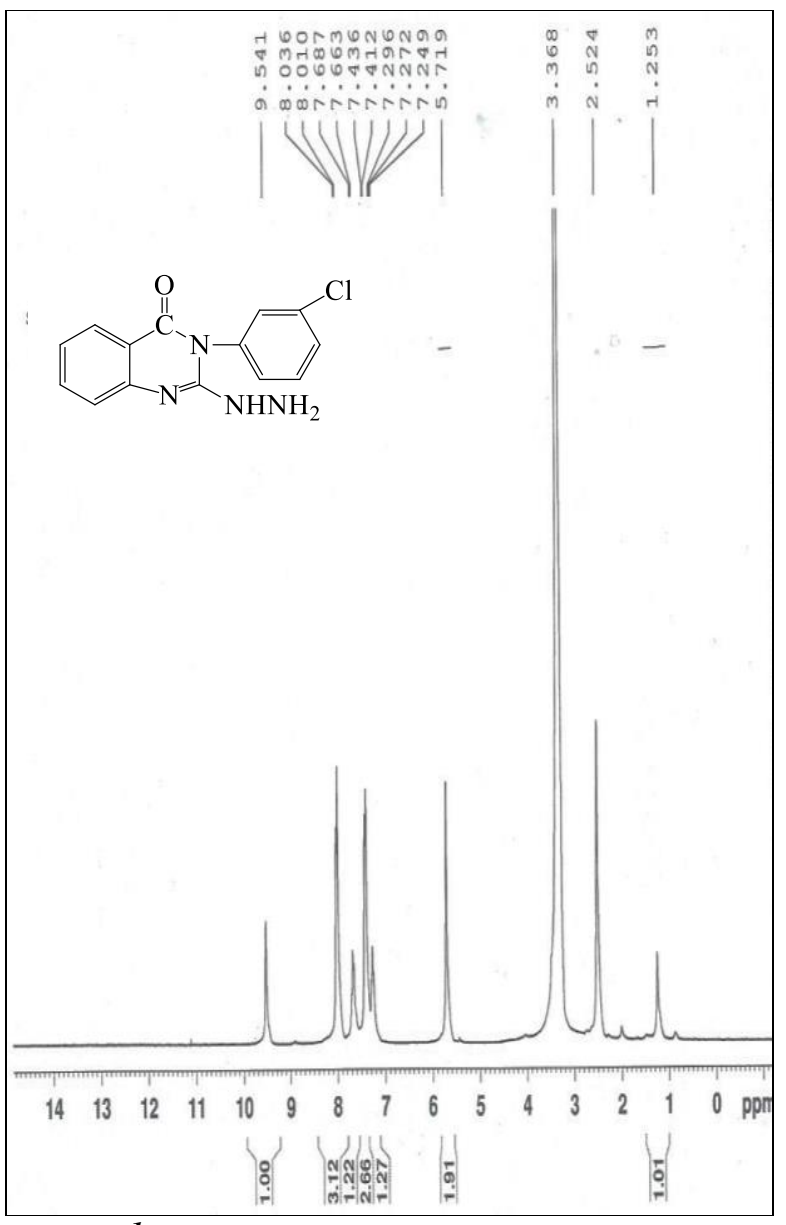

Fig.(9) ${ }^{1} \mathrm{H}$-NMR spectrum of compound (4a).

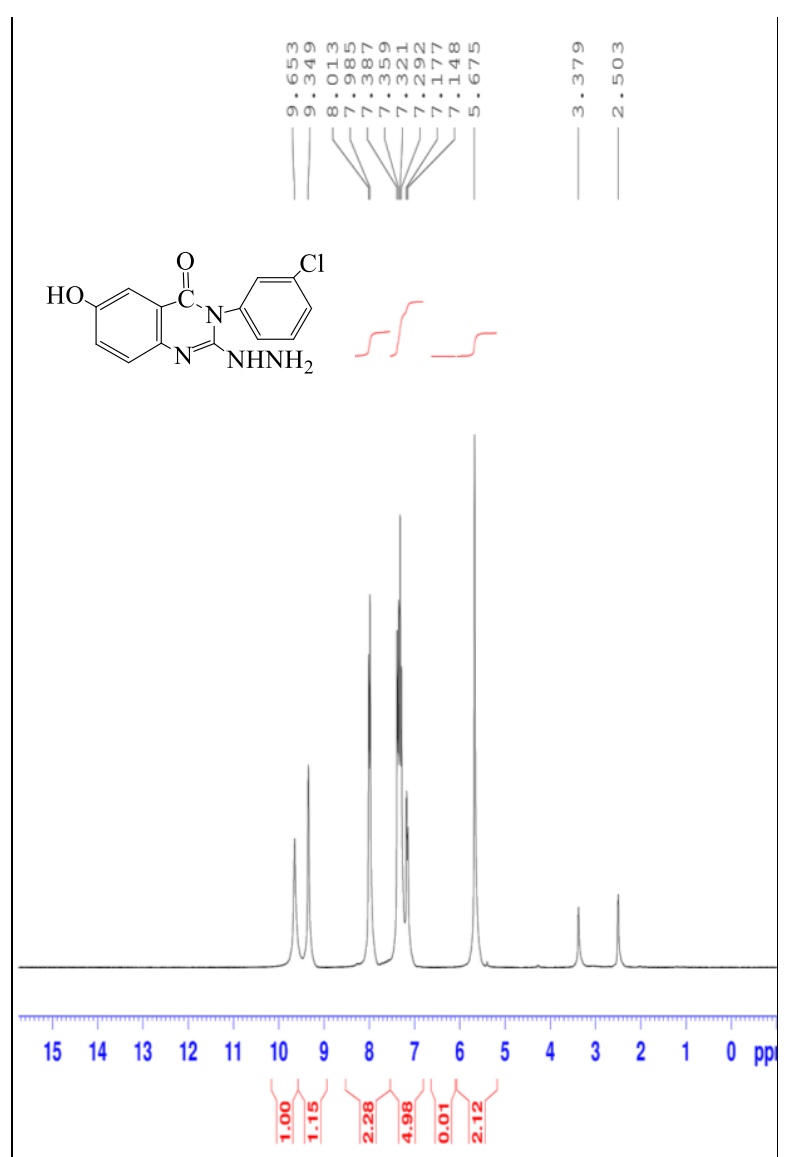

Fig.(10) ${ }^{1} \mathrm{H}$-NMR spectrum of compound (4b).

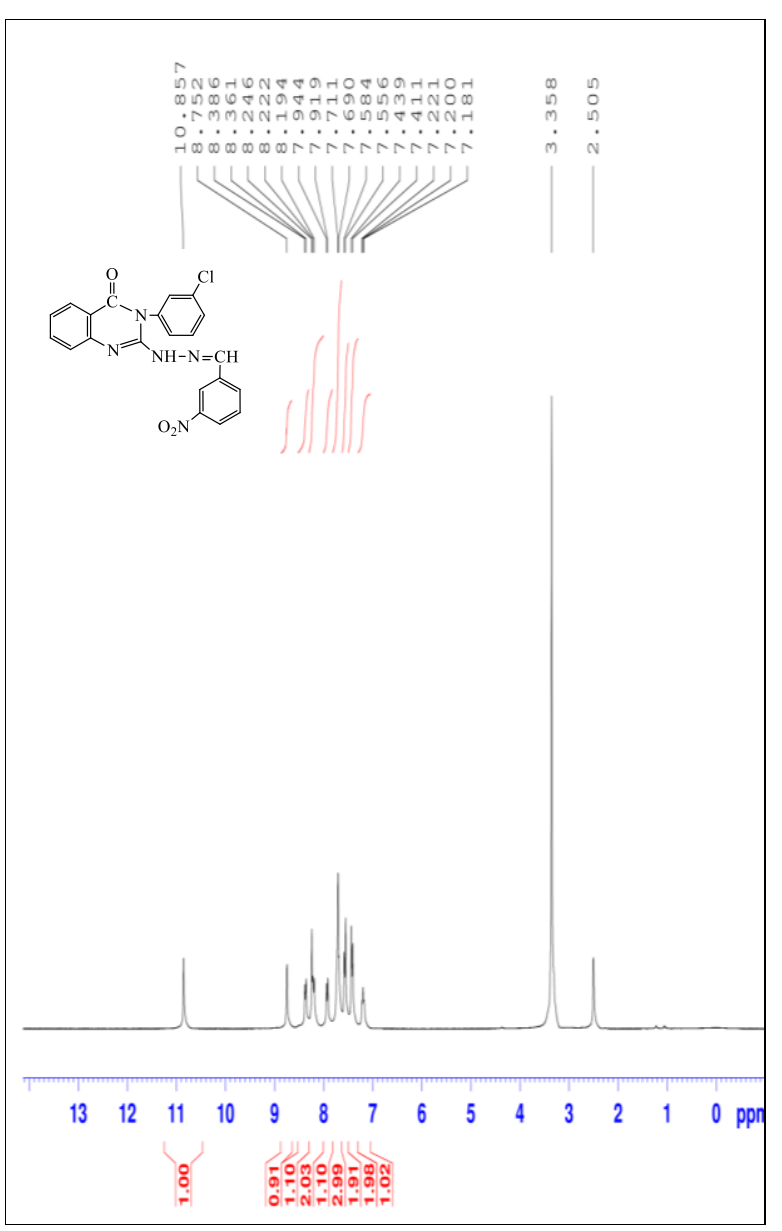

Fig.(11) ${ }^{1} \mathrm{H}-\mathrm{NMR}$ spectrum of compound (6a).

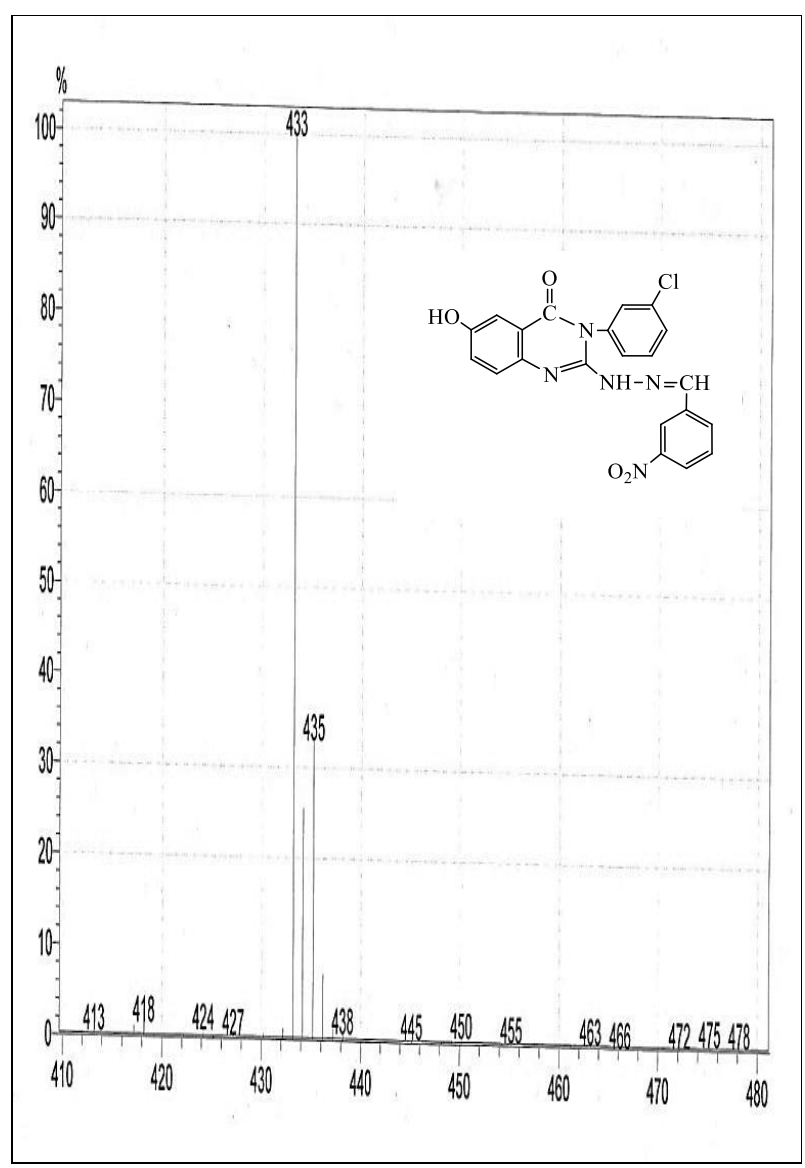

Fig.(12) ${ }^{1} H$-NMR spectrum of compound (6b). 
Souad J. Lfta

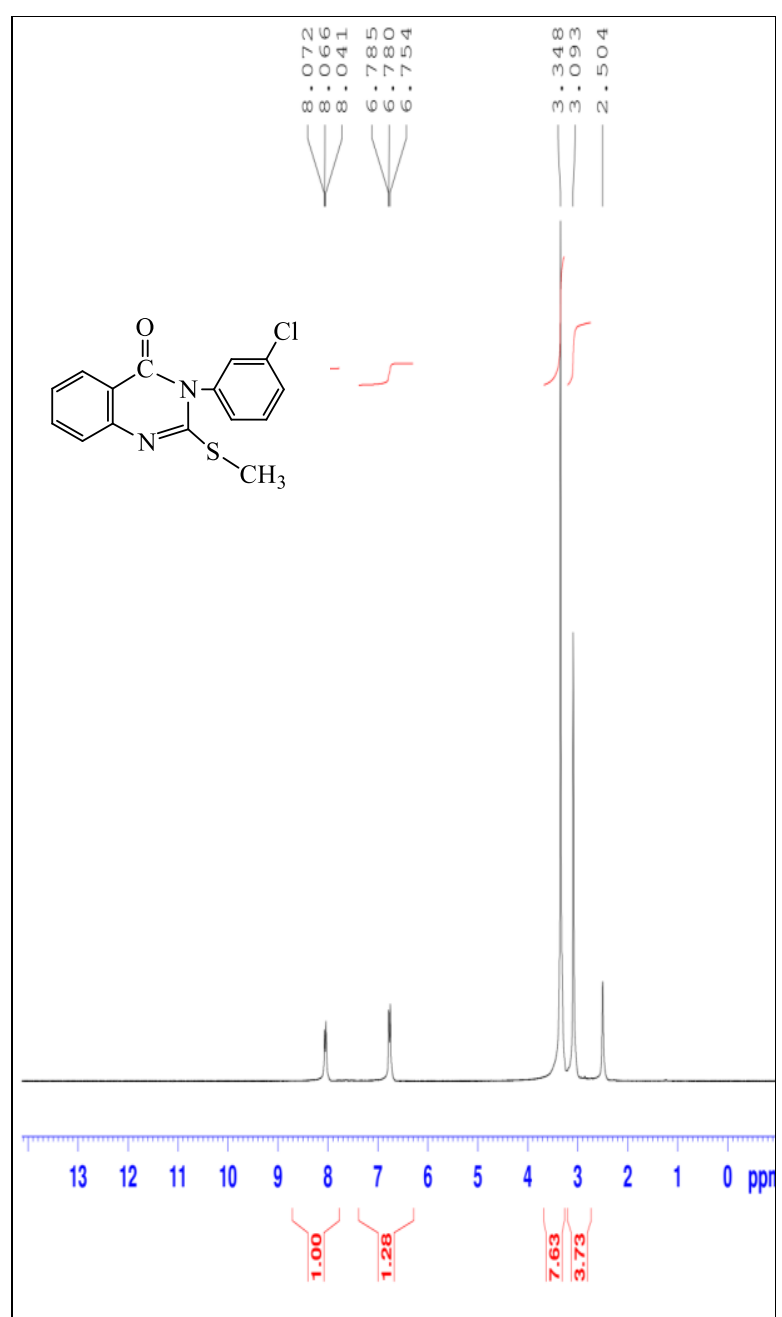

Fig. (13) ${ }^{1} \mathrm{H}$-NMR spectrum of compound (7a).

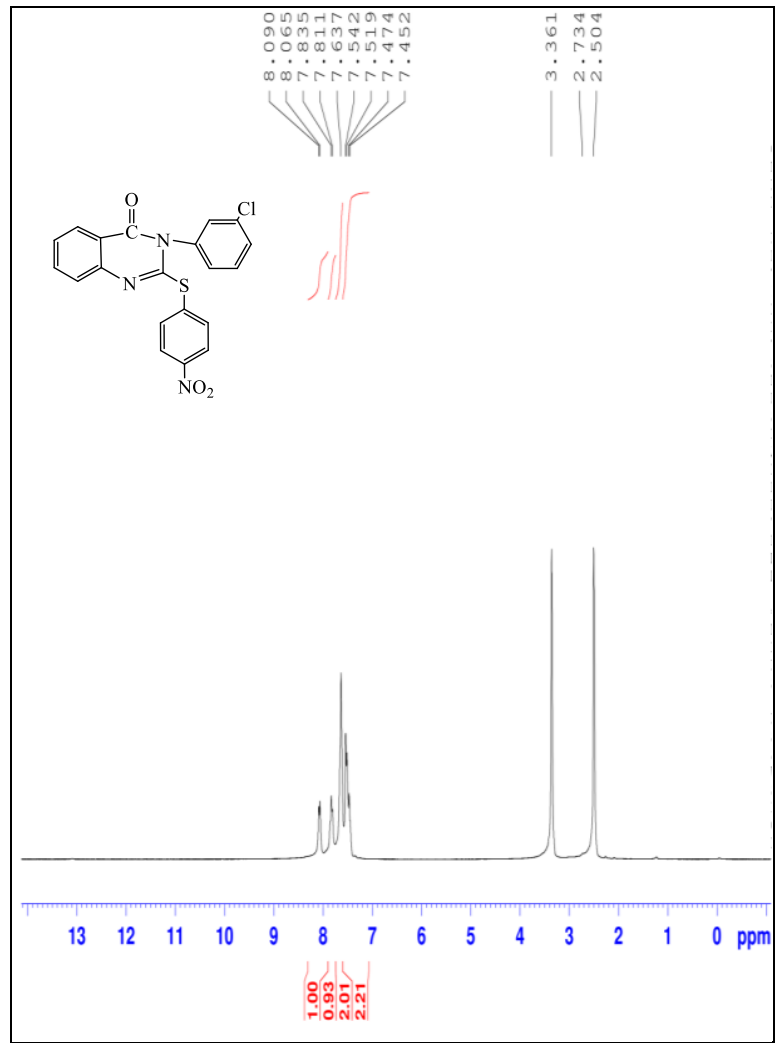

Fig.(14) ${ }^{1}$ H-NMR spectrum of compound (7b).

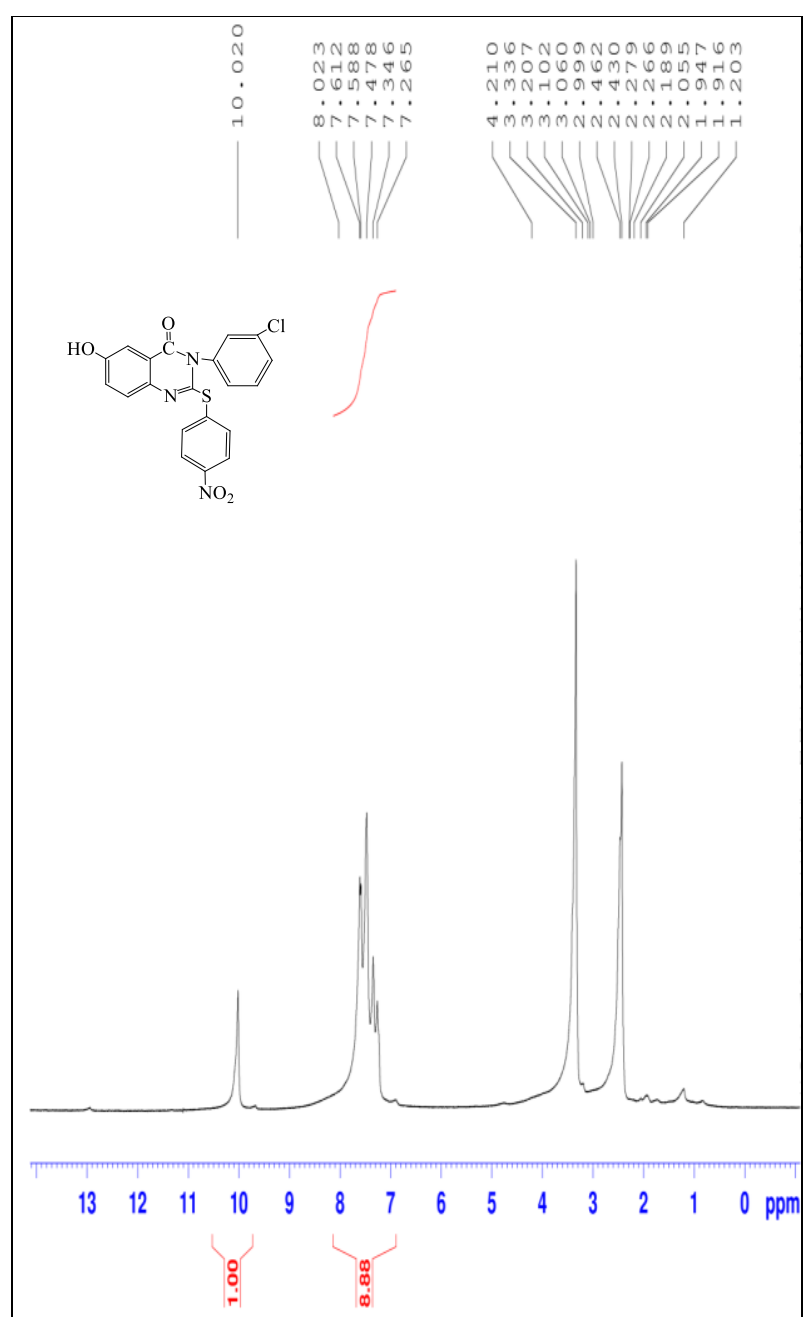

Fig. (15: $)^{1} H-N M R$ spectrum of compound (8b).

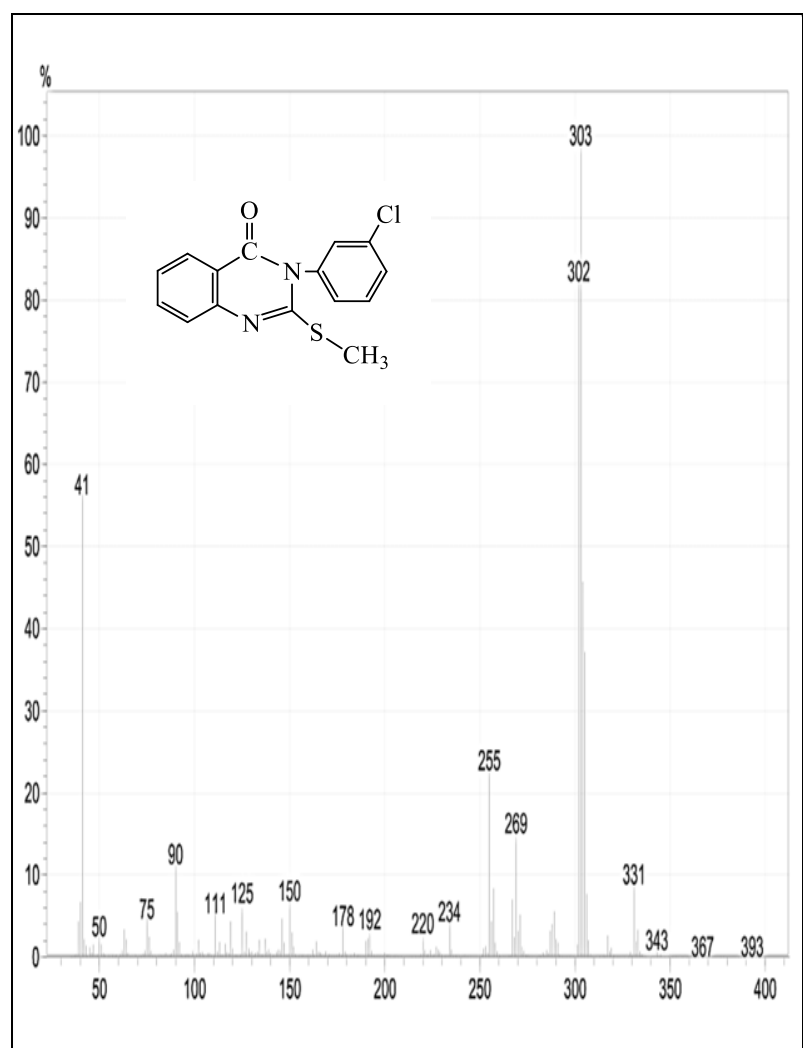

Fig. (16) MASS spectrum of compound (7a). 


\section{References}

[1] Saravanan G., Alagarsamy V. and Parkash C., "Design, synthesis and anticonvulsant activities of novel 1-(substituted/ unsubstituted benzylidene)-4-(4-(6,8dibromo-2-(methyl/phenyl)-4-oxoquinazolin3(4H)-yl)phenyl) semicarbazide derivatives", Bioor. Med. Chem. Lett., 22(9), 3072-3078, 2012.

[2] Abbas S., Awadallah F., Ibrahin N., Said E. and kamel, G., "New quinazolinonepyrimidine hydride: synthesis, antiinflammatory, and ulcerogenicity studies", Eur. J.Med. Chem., 53, 141- 149, 2012.

[3] El-Azab A. and EI-Tahir K., "Design and synthesis of novel 7-aminoquinazoline derivatives: antitumor and anticonvulsant activities", Bioorg. Med. Chem.Lett., 22, 1879-1885, 2012.

[4] Aly M., Mohameda A.; El - Bayouki A.; Basyouni M. and Abbas Y., "Synthesis of some new 4(3H)-quinazoline -2carboxaldehydethiosemi carbazones and their anticonvulsant, analgesic, cytotoxic and antimicrobial activities -part-1, Eur. J. Med. Chem., 45, 3365 -3373, 2010.

[5] Giri S., Thaker M., Giordano T., Chen B., Nuthalapaty S., Vasu K. and Sudarsanam V., "Synthesis and evalution of NF-kappa B, AP-1mediated transcription and elf4Emediated translationd activation: inhibitors of multi-pathways in volve in cancer", Eur. J. Med. Chem., 45, 35583563, 2010.

[6] Eguchi H., Tasaka H., Hirai S., Takaya Y., Lwabuchi Y., Oie H., Hatakeyama S., Kim H., Wataya Y. and Oshima Y., "Synthesis of optically active vasicinone based on intramdecular Aza-witting reaction asymmetric oxidation", J.Org. Chem., 61, 7316, 1996.

[7] Bergman J. and Brynoef A., "Science of synthesis: houben-weyl methods of molecular transformation", Tetrahedron, 46, 1295, 1990.

[8] Bergman J., "Quadrature domains and their applications", J. Chem. Res.(s), 224, 1997.

[9] John S., "In supplements to the 2nd edition of rodd's chemistry of carbon compounds", M.F. Ansell. ed, Elsevier: Amsterdam, 223, 1955.
[10] Kunes J., Bazant J., Pour M., Waisser K., Slosarek M.and Janata J. "Quinazoline derivatives with antitubercular activity", IL Farmaco, 55, 725-729, 2000.

[11] Gemma S., Kukreja G., Fattorusso C., Persico M., Romano M., Altarelli M., Savini L., Campiani G., Fattorusso E. and Basilico N., "Synthesis of N1-arylideneN2-quinoly-andN2-acrydinylhydrazones as potent antimalarial agents active againstCQ-resistant P. fbciparum strains", Bioorg. \& Med. Chem. Lett., 16(20), 5384-5388, 2006.

[12] Caffrey C., Schanz M., Njinkeng J., Brush M., Hansell E., Cohen F., Flaherty T., Mckerrow J. and Steverding D., "Nuevos avances en el desarrollo del tratamiento etiologico de la enformedad de chagas", Int. J. Antimicrob., 19, 227-231, Ag. 2002.

[13] Sheldrick G., "2-(4-chloro anilino)-3-(2hydroxyethyl) quinazolin-4(3H)-one", Acta Cryst., A64, 112-122, 2008.

[14] Al-Omar M., Abdel- Hamide S., AlKhamees H. and El-Subbagh H., "Synthesis and biological screening of some new substituted- 3H-Quinazolin-4-one analogs as antimicrobial agents", Saudi pharmaceutical Journal, 12, 2-3 July, 2004.

[15] Mahmoud M., Abou-Elmagd W., Abdel wahab S. and El-Sayed S., "Synthesis and spectral characterization of novel 2,3disubstituted Quinazolin-4 $(3 \mathrm{H})$ one derivatives", American Journal of organic Chemistry, 2(1): 1-8, 2012.

[16] SAEED A., MAHOOD Sh. and FLÖRKE Ul., "Synthesis and characterization of new (E)-N'-(substituted benzylidene)-2-(3-(2-methyl) -4-oxo-3,4dihydroquinazolin-2-ylthio) acetohydrazides", Turkish. J. Chem., 38, 275-287, 2014.

[17] Silverstein R., "Spectrometric indentification of organic compounds", 4th.ed, 1, 1981.

[18] Shriner R., Fuson R., Curtin D. and Morill, T., "The systematic identification of organic compounds", a laboratory manual. 6th. ed, QD.261.S5, 1979. 
[19] Sevgi F. and Bedük D., "Synthesis and antimicrobial activity of novel glyoximes containing quinoline moiety", World Applied Sciences Journal, 19(2):192-197, 2012.

[20] Khan M., Miller K., Rainsford D. and Zhou Y., "Synthesis and antimicrobial activity of novel substituted ethyl 2(quinolin-4-yL)-propanoates", Molecules Journal, 18, 3227-3240, 2013.

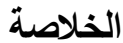

في هذا البحث نم تحضير بعض من مشتقات

الكوينازولين ثنائية التعويض في -3,2- من تفاعل حامض ندان

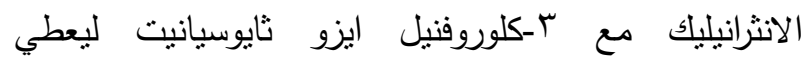

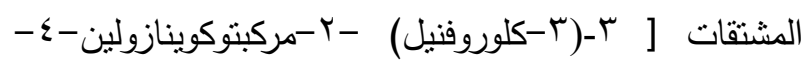

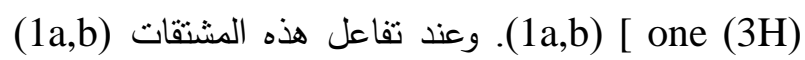

مع كلورو اثيل اسيتات بوجود كاربونات البوتاسيوم اعطت

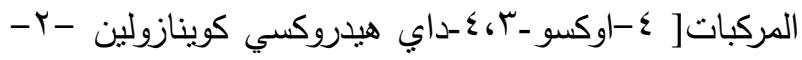

يل) ثايو اسيتات (2a,b). أن تفاعل المشتقات (2a,b) مع هين

الهيدرازين المائي اعطت مشتقات الاسيتوهيدرازايد (3a,b).

عند تفاعل مشتقات الاسيتوهيدرازايد (3a,b) مع الالديهايدات

الاروماتية اعطت قواعد شيف (5a,b). ان تفاعل المشتقان

(1a,b)

(4a,b)

مشتقات قواعد شيف (6a,b). عند تفاعل المشتقان (1a,b)

مع هاليدات الالكيل في الاسينون تكونت المشتقات (7a,b),

اما عند تفاعلها مع الهاليدات الاروماتية في ثنائي مثنيل

الاميد فقد تكونت المشتقات (8a,b). لقد شخصت نراكيب

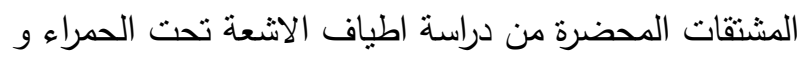

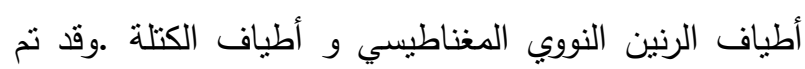

تقييم الفعالية ضد البكتريا للمركبات a-b (1,5,6,7). 